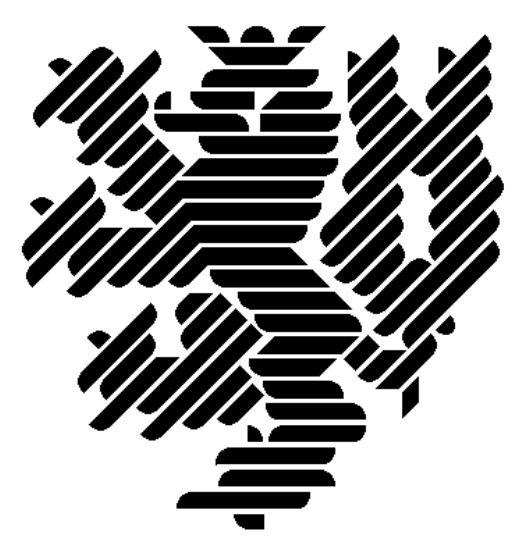

Bergische Universität Wuppertal

Fachbereich Mathematik und Naturwissenschaften

Lehrstuhl für Angewandte Mathematik

und Numerische Mathematik

Lehrstuhl für Optimierung und Approximation

Preprint BUW-AMNA-OPAP 10/07

Anton Mezentsev, Anton Pomelnikov and Matthias Ehrhardt

\title{
Efficient Numerical Valuation of Continuous Installment Options
}

April 2010

http://www.math.uni-wuppertal.de 


\title{
Efficient Numerical Valuation of Continuous Install- ment Options
}

\author{
Anton Mezentsev ${ }^{1, *}$, Anton Pomelnikov ${ }^{1}$ and Matthias Ehrhardt ${ }^{2}$ \\ ${ }^{1}$ IDE, Halmstad University, Box 823, 301 18, Halmstad, Sweden \\ ${ }^{2}$ Lehrstuhl für Angewandte Mathematik und Numerische Analysis, Fachbereich C \\ - Mathematik und Naturwissenschaften, Bergische Universität Wuppertal, Gaußstr. \\ 20, 42119 Wuppertal, Germany
}

\begin{abstract}
In this work we investigate the novel Kryzhnyi method for the numerical inverse Laplace transformation and apply it to the pricing problem of continuous installment options. We compare the results with the one obtained using other classical methods for the inverse Laplace transformation, like the Euler summation method or the Gaver-Stehfest method.
\end{abstract}

AMS subject classifications: 65M10, 78A48

Key words: Installment options, Black-Scholes equation, numerical inverse Laplace transform, Gaver-Stehfest method, Euler summation method, optimal stopping problem, non-monotonic stopping boundary

\section{Introduction}

Starting from the ancient age people tried to hedge their trading risks. We can find the predecessors of trading options looking at the history of ancient Rome, Phoenicia, Greece. With time passing options became more and more popular, drawing not only the hedgers, but the speculators also. In 1848, the new page of options' history was written as the Chicago Board of Trade (CBOT) was set up and the options started being traded officially. Developing rather slowly the option market then got into the boom in the end of 1960's - middle 1970's caused by the opening of the Chicago Board Options Exchange (CBOE) and the appearing of the well-known Black-Scholes model. This was the time when the modern history of the options started. Since that moment the interest in options was growing: the volumes of trading have increased, variety of new options types has appeared.

What are options today? Basically, options represent the right to buy (sell) the asset by a predetermined price at a certain date. The predetermined price in the option

*Corresponding author.

Email: a.g.mezentsev@gmail.com (Anton Mezentsev), pomelnikov.anton@gmail.com (Anton Pomelnikov), ehrhardt@math.uni-wuppertal.de (Matthias Ehrhardt) 
contract is known as the exercise price or strike price, the date of contract expiring is called the exercise date or maturity. At the exercise date the option holder has the right to acquire (sell) an underlying asset by the strike price or spot price regardless what is more favorable to him. The holder has to pay for this right, that is why the option has a certain price, the so-called option premium. Options where the holder buys the asset is known as call option. If the holder sells the asset the option is called put option. Talking about the time of exercising, options might be of European or American type. While European options can be exercised only at maturity, American options can be exercised at any moment up to the maturity.

This work is devoted to one of the quite recently emerged options, the so-called installment option. Installment option is a financial derivative where the small initial premium is paid up-front and the other part of the premium is divided into the installments to be paid during the lifetime of the contract up to maturity. At each installment date the investor has the right to decide if he continues to pay for the contract or he terminates paying, allowing the option to lapse.

Nowadays, installment options are rather widely traded in the financial markets. They possess significant advantage over other options. It is the possibility to stop paying the premiums before the option is expired. Thanks to this property the companies carrying out the policy of investments can reduce their losses. Moreover, taking in mind the nature of the installment options we can find a number of other contracts similar to them: some life insurance contracts and capital investment projects might be considered as installment options (see Dixit and Pindyck [12]). Thomassen and Van Wouwe [29] applied the installment option in pharmacy comparing the development of a new drug, evolving 6 stages, with a 6-variate installment option; MacRae [23] modeled the employee stock option as an installment option, etc.

As for the way of payments there exist 2 types of installment options: discrete and continuous. The discrete installment options are investigated concisely in the works of Karsenty and Sikorav [19], Davis et al. [10,11], Ben-Ameur et al. [4] and Griebsch et al. [17].

The treatment of the continuous installment options is more complicated. There only exists a few related works. Ciurlia and Roko [6] studied the American case applying the multipiece exponential function (MEF) method to derive an integral form of the option's value. Their applied technique suffers from a serious drawback, since the MEF method generates a discontinuity in the optimal stopping and early exercise boundaries. Alobaidi [2] analyzed the European case using the Laplace transformation to solve the free boundary problem. However, the method used is rather specific and not suitable for a numerical computation.

In the current paper we focus on the valuation of the continuous installment options starting from the paper of Kimura [20], who successfully applied the Laplace transformation method to valuate these options numerically. Our goal is to revisit and correct Kimura's results and introduce the new Kryzhnyi method of inverse Laplace transformation to the field of option pricing in order to valuate the continuous installment options and their Greeks. 
The outline of this work is as follows. In Section 2 we briefly introduce the installment options, consider their essentials and features. In Section 3 we describe the model and formulate the valuation problem of both call and put continuous installment options. In Section 4 we describe the Laplace-Carson transformation method, the Gaver-Stehfest and the Kryzhnyi methods of the inverse Laplace transformation, used for the numerical valuation of the installment options. The theoretical results of the valuation of the stopping boundary, the values of the options and their Greeks are included in Section 5, the numerical results are presented in Section 6. Finally, we draw our conclusions in Section 7.

\section{Introduction to Installment Options}

The definition of installment options can be formulated in the following way: the option where the premium is divided into different parts and is paid during the option lifetime. Every installment date presents the moment when the holder takes the decision either to continue to pay the premiums or allow the contract to lapse. The total premium of the installment option is always higher than the vanilla options premium. This property can be explained by the additional opportunities to terminate the contract without paying the whole sum of the premium.

The installment option is interesting for the investors who are ready to overpay for the advantage to terminate the payments and reduce the losses if their investment position goes wrong.

Dealing with installment options we can separate two cases of the installment payments: discrete and continuous.

- Discrete case means that the installment option has a finite number of exercise dates, e.g. 3, 6, 8. At each of these dates the investor can terminate paying, allowing the option to lapse.

- Continuous case means that the holder pays a stream of installments at a given rate per unit time. In real life it looks like accumulating the premium sum by a certain continuous rate, afterwards paid by the holder in the case of exercising. The holder has the choice to stop the contract at any time before the maturity. This opportunity turns the valuation of the installment options into a free boundary problem. For the continuous case there exist two types of installment options: European and American.

We present the scheme (Figure 1) distinguishing types of installment options.

In the current paper we consider the case of the European continuous installment options only, so referring to them further as "continuous installment options". 


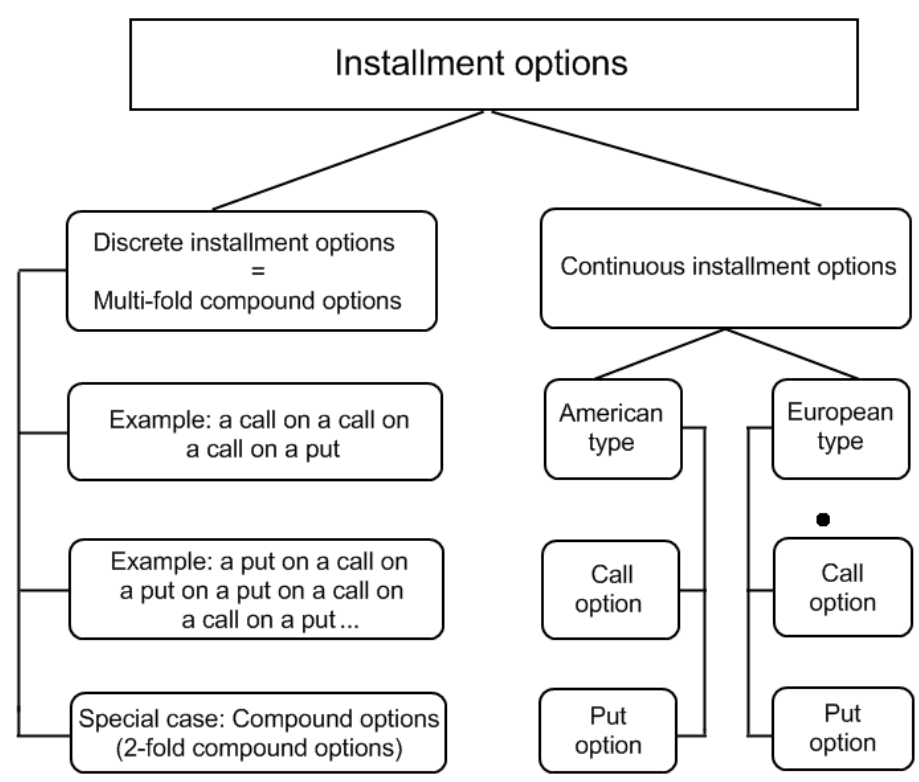

Figure 1: The classification of installment options.

\section{Analytical Valuation of the Continuous Installment Options}

\subsection{The Model}

Pursuing the aim of the work - to valuate the continuous installment options - we decide to base our considerations on the classical Black-Scholes model.

Assume that the price of the underlying asset $S_{t}$ obeys the geometric Brownian motion described by the stochastic differential equation

$$
\frac{d S_{t}}{S_{t}}=\mu d t+\sigma d W_{t}
$$

where $\mu=(r-\delta), r$ and $\delta$ denote the interest rate and the continuous dividend yield respectively. $\sigma$ is volatility and $d W_{t}$ is a standard Brownian motion on a risk-neutral probability space. The value $V_{t}=V\left(t, S_{t} ; q\right)$ of the continuous installment option depends on the time $t$, the spot price of the underlying $S_{t}$ and the continuous installment rate $q$. In time $d t$ the holder pays the premium $q d t$ to continue the contract.

Using Itô's Lemma to derive the dynamics for the value of a continuous installment option, we obtain

$$
d V_{t}=\left(\frac{\partial V_{t}}{\partial t}+(r-\delta) S_{t} \frac{\partial V_{t}}{\partial S}+\frac{1}{2} \sigma^{2} S_{t}^{2} \frac{\partial^{2} V_{t}}{\partial S^{2}}-q\right) d t+\sigma S_{t} \frac{\partial V_{t}}{\partial S} d W_{t}
$$

We take a portfolio that includes the continuous installment option and $-\Delta$ amount of the underlying asset

$$
\Pi_{t}=V_{t}-\Delta S_{t}
$$


with dynamics

$$
d \Pi_{t}=d V_{t}-\Delta d S_{t}-\Delta\left(S_{t} \delta d t\right)
$$

Plugging (3.1) and (3.2) into (3.3), we obtain

$$
\begin{aligned}
d \Pi_{t}= & \left((r-\delta)\left(\frac{\partial V_{t}}{\partial S}-\Delta\right)+\frac{\partial V_{t}}{\partial t}+\frac{1}{2} \sigma^{2} S_{t}^{2} \frac{\partial^{2} V_{t}}{\partial S^{2}}-q-\Delta S_{t} \delta\right) d t \\
& +\sigma S_{t}\left(\frac{\partial V_{t}}{\partial S}-\Delta\right) d W_{t} .
\end{aligned}
$$

To remove the risk of uncertainty we select $\Delta=\frac{\partial V_{t}}{\partial S}$. This choice turns the portfolio riskless now and it has to yield the return $r$ to avoid any arbitrage opportunities

$$
r\left(V_{t}-\frac{\partial V_{t}}{\partial S} S_{t}\right)=\left(\frac{\partial V_{t}}{\partial t}+\frac{1}{2} \sigma^{2} S_{t}^{2} \frac{\partial^{2} V_{t}}{\partial S^{2}}-q-\frac{\partial V_{t}}{\partial S} S_{t} \delta\right) .
$$

Finally, we get an inhomogeneous Black-Scholes partial differential equation (PDE) for the valuation of the continuous installment options

$$
\frac{\partial V_{t}}{\partial t}+(r-\delta) S_{t} \frac{\partial V_{t}}{\partial S}+\frac{1}{2} \sigma^{2} S_{t}^{2} \frac{\partial^{2} V_{t}}{\partial S^{2}}-r V_{t}=q
$$

Here, $q$ should be strictly positive; if it is equal to zero the Black-Scholes PDE turns into the homogeneous type.

\subsubsection{The Call Case}

We consider the European installment call option $c\left(t, S_{t} ; q\right)$ with the maturity $T$ and the exercise price $K$. The payoff at the maturity is $\max \left(S_{T}-K, 0\right)$. The opportunity to terminate the contract at any time $t \in[0, T]$ makes the valuation of a continuous installment option an optimal stopping problem. In other words, we need to find such points $\left(t, S_{t}\right)$ that the termination of the option is optimal.

We consider the domain $\mathcal{D}=[0, T] \times[0,+\infty]$ and denote the stopping region and the continuation region by $\mathcal{S}$ and $\mathcal{C}$, respectively. Then, the stopping region is given by

$$
\mathcal{S}=\left\{\left(t, S_{t}\right) \in \mathcal{D} \mid c\left(t, s_{t} ; q\right)=0\right\},
$$

the optimal stopping time $\tau_{c}^{*}$ is defined by

$$
\tau_{c}^{*}=\inf \left\{u \in[t, T] \mid\left(u, S_{u}\right) \in \mathcal{S}\right\} .
$$

Being the complement of $\mathcal{S}$ in $\mathcal{D}$, the continuation region $\mathcal{C}$ has the representation

$$
\mathcal{C}=\left\{\left(t, S_{t}\right) \in \mathcal{D} \mid c\left(t, S_{t} ; q\right)>0\right\} .
$$

The boundary separating the two regions $\mathcal{S}$ and $\mathcal{C}$ is called stopping boundary, and is defined by

$$
\underline{S}_{t}=\inf \left\{S_{t} \in[0,+\infty) \mid c\left(t, S_{t} ; q\right)>0\right\} .
$$


The stopping boundary $\left(\underline{S}_{t}\right)_{t \in[0, T]}$ is essentially the lower critical asset price below which it is necessary to terminate the contract.

In the continuation region $\mathcal{C}$, where $S>\underline{S}_{t}$, the call value $c(t, S ; q)$ can be determined from the inhomogeneous Black-Scholes PDE

$$
\frac{\partial c}{\partial t}+(r-\delta) S \frac{\partial c}{\partial S}+\frac{1}{2} \sigma^{2} S^{2} \frac{\partial^{2} c}{\partial S^{2}}-r c=q
$$

supplied with the boundary conditions

$$
\lim _{S \rightarrow \underline{S}_{t}+0} c(t, S ; q)=0, \quad \lim _{S \rightarrow \underline{S}_{t}+0} \frac{\partial c}{\partial S}=0, \quad \lim _{S \rightarrow \infty} \frac{\partial c}{\partial S}<\infty,
$$

and the terminal condition

$$
c(T, S ; q)=\max (S-K, 0) .
$$

The following integral representation is the value function of the continuous installment call option, cf. [20]:

$$
c\left(t, S_{t} ; q\right)=c\left(t, S_{t}\right)-q \int_{t}^{T} e^{-r(u-t)} \Phi\left(d_{-}\left(S_{t}, \underline{S}_{u}, u-t\right)\right) d u,
$$

where

$$
d_{ \pm}(a, b, \tau)=\frac{\log (a / b)+\left(r-\delta \pm \frac{1}{2} \sigma^{2}\right) \tau}{\sigma \sqrt{\tau}} .
$$

In (3.5) $c\left(t, S_{t}\right)=c\left(t, S_{t} ; 0\right)$ is the value of the European vanilla call option given by

$$
c\left(t, S_{t}\right)=S_{t} e^{-\delta(T-t)} \Phi\left(d_{+}\left(S_{t}, K, T-t\right)\right)-K e^{-r(T-t)} \Phi\left(d_{-}\left(S_{t}, K, T-t\right)\right) .
$$

From expression (3.5) we can easily see that the price of the continuous installment option can be represented as the difference between the European vanilla call option and the expected discounted value of the installment premiums along the optimal stopping boundary. Actually, due to the boundary conditions, the optimal stopping boundary $\left(\underline{S}_{t}\right)_{t \in[0, T]}$ obeys the integral equation

$$
c\left(t, \underline{S}_{t}\right)-q \int_{t}^{T} e^{-r(u-t)} \Phi\left(d_{-}\left(\underline{S}_{t}, \underline{S}_{u}, u-t\right)\right) d u=0 .
$$

\subsubsection{The Put case}

We proceed in this case analogously to the approach for the call case. Let us consider the European installment put option $p\left(t, S_{t} ; q\right)$ with the maturity date $T$ and the exercise price $K$. Now $\bar{S}_{t}$ denotes the upper asset price above which the holder has to terminate the contract. The stopping boundary $\left(\bar{S}_{t}\right)_{t \in[0, T]}$ also divides $\mathcal{D}$ into 2 regions: a continuation region $\mathcal{C}=\left\{\left(t, S_{t}\right) \in[0, T] \times\left[0, \bar{S}_{t}\right)\right\}$ and a stopping region $\mathcal{S}=\left\{\left(t, S_{t}\right) \in[0, T] \times\left[\bar{S}_{t}, \infty\right)\right\}$. 
In the continuation region $\mathcal{C}$, where $S<\bar{S}_{t}$, the value of the continuous installment put option $p(t, S ; q)$ can be determined from the inhomogeneous Black-Scholes PDE

$$
\frac{\partial p}{\partial t}+(r-\delta) S \frac{\partial p}{\partial S}+\frac{1}{2} \sigma^{2} S^{2} \frac{\partial^{2} p}{\partial S^{2}}-r p=q,
$$

supplied with the boundary conditions

$$
\lim _{S \rightarrow \bar{S}_{t}-0} p(t, S ; q)=0, \quad \lim _{S \rightarrow \bar{S}_{t}-0} \frac{\partial p}{\partial S}=0, \quad \lim _{S \rightarrow+0} \frac{\partial p}{\partial S}<\infty,
$$

and the terminal condition

$$
p(T, S ; q)=\max (K-S, 0) .
$$

The value function of the continuous installment put option has the following integral expression [20]:

$$
p\left(t, S_{t} ; q\right)=p\left(t, S_{t}\right)-q \int_{t}^{T} e^{-r(u-t)} \Phi\left(-d_{-}\left(S_{t}, \bar{S}_{u}, u-t\right)\right) d u,
$$

where $p\left(t, S_{t}\right)=p\left(t, S_{t} ; 0\right)$ denotes the value of the European vanilla put option

$$
p\left(t, S_{t}\right)=K e^{-r(T-t)} \Phi\left(-d_{-}\left(S_{t}, K, T-t\right)\right)-S_{t} e^{-\delta(T-t)} \Phi\left(-d_{+}\left(S_{t}, K, T-t\right)\right) .
$$

In order to compute the values of these options and, hence for this purpose, the optimal stopping boundaries, we need to apply a numerical approach in the sequel.

\subsection{A Decomposition of the Total Premium}

Griebsch et al. [17] proved that the premium sum of the continuous installment option is equal to the respective European vanilla option plus the right to leave at any time at a pre-determined rate. In other words, they observed that the total premium of the continuous installment call option equals the European vanilla call option plus an American put option on this European call with floating strike price

$$
c\left(t, S_{t} ; q\right)+K_{t}=c\left(t, S_{t}\right)+P\left(t, S_{t} ; q\right),
$$

where $K_{t}=\frac{q}{r}\left(1-e^{-r(T-t)}\right)$ is the discounted sum of the premiums not to be paid if the contract is terminated at the moment $t$, and for the set $\mathcal{S}_{t, T}$ of stopping times with values in $[t, T]$ (a.s.)

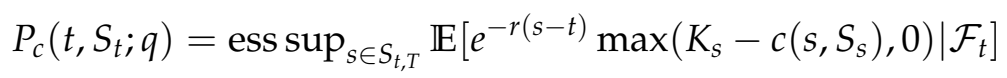

is the value of the American compound put option with the maturity at $T$ written on the European vanilla call option. Let us emphasize the fact that this decomposition is needed to obtain the Greeks formulas for the continuous installment options. 


\section{Methods}

\subsection{The Laplace Transform}

Integral transforms are very actively used in various ways to solve problems of the applied mathematics. Among other things Cohen [7] presents a couple of applications of the Laplace transform in a heat conduction in a rod, laser anemometry and, particularly, exotic options valuing.

Definition 1 (Laplace transformation). If for a function $f: \mathbb{R}^{+} \rightarrow \mathbb{C}, f: t \mapsto f(t)$ there exists a $\sigma \in \mathbb{R}$, such that the integral the Laplace transform of the function $f(t)$ is defined by

$$
\mathcal{L}\{f\}(\lambda):=F(\lambda):=\int_{0}^{\infty} f(t) e^{-\lambda t} d t,
$$

exists for all $\lambda \in \mathbb{C}$ with $\operatorname{Re} \lambda>\sigma$, then $F(\lambda)$ is the Laplace transformation of $f$.

Remark 1. If $f: \mathbb{R}^{+} \rightarrow \mathbb{C}, f: t \mapsto f(t)$ is of exponential order, i.e. for some $\gamma \in \mathbb{R}$

$$
\sup _{t>0} \frac{|f(t)|}{e^{\gamma t}}<\infty
$$

then the Laplace transform (4.1) exists for all $\lambda>\gamma$ and is inifinitely differentiable with respect to $\lambda$ for $\lambda>\gamma$.

We will apply the Laplace transform (4.1) to the Black-Scholes PDE (with the two variables time and asset price) thus reducing it to an ordinary differential equation (ODE) with respect to the asset price. Note that in our work we will consider a slight modification, the Laplace-Carson transformation.

Definition 2 (Laplace-Carson transformation). For the same assumptions as above the Laplace-Carson transformation of the function $f(t)$ is defined by

$$
\mathcal{L C}\{f\}(\lambda):=\lambda \int_{0}^{\infty} f(t) e^{-\lambda t} d t .
$$

Let us note that the only reason for using (4.3) instead of (4.1) is that it generates more simple formulas for the transformed values.

\subsection{The Inverse Laplace Transform}

For a function $F(\lambda)=\mathcal{L}\{f\}(\lambda)$ in the transformed $\lambda$-space the inverse of the Laplace transformation is given by the Bromwich integral

$$
\mathcal{L}^{-1}\{F\}(t)=f(t)=\frac{1}{2 \pi i} \int_{\gamma-i \infty}^{\gamma+i \infty} F(\lambda) e^{\lambda t} d \lambda
$$


where $\gamma$ is a vertical contour in the complex plane chosen such that all singularities of $F(\lambda)$ are to the left of it. Note that according to Lerch's theorem [7] the Laplace transform is unique for every continuous function $f(t)$.

Now if we have an ODE solution for the corresponding transformed PDE, and an exact formula for determining $\mathcal{L}^{-1}\{F\}(t)$ we can easily produce a representation of the solution for our PDE. However, it is well-known that the the numerical inverse Laplace transform is an inherently ill-posed problem and thus requires special considerations.

\subsection{The Numerical Inverse Laplace Transform}

\subsubsection{The Post-Widder formula}

Post and Widder [26,32] presented the original function $f(t)$ as a limit of some sequence, involving $F^{(n)}$, the $n$-th derivative of $F(\lambda)$, on the real axis, which is more convenient for the numerical computation of the inverse Laplace transform than trying to compute the integral on the complex plane. The result is formulated in the following theorem.

Theorem 1 (Post and Widder theorem [7])). If for a continuous function $f(t)$ the integral

$$
F(\lambda)=\int_{0}^{\infty} e^{-\lambda t} f(t) d t,
$$

converges for every $\lambda>\gamma$ (sufficient for this is e.g. the growth condition (4.2)), then

$$
f(t)=\lim _{n \rightarrow \infty} \frac{(-1)^{n}}{n !}\left(\frac{n}{t}\right)^{n+1} F^{(n)}\left(\frac{n}{t}\right),
$$

where $F^{(n)}$ denotes the $n$-th derivative of $F(\lambda)$.

There are two major problems of using this formula (4.5) for the numerical inverse Laplace transform. The first problem is that differentiating $F(\lambda)$ a large number of times can be a big obstacle if $F(\lambda)$ is a complicated function, even if symbolic Maple or Mathematica differentiating routines are used. Besides, it is well-known that high order derivatives are sensible to the round-off errors causing thereby instabilities. Hence, this approach seems impractical for most purposes. The second problem is that the convergence to the limit is very slow. However, the convergence can be speeded up using an appropriate extrapolation technics, cf. [1,30]

We remark that this elementary inversion formula (4.5) yield, as a side effect, a characterization of non-negative solutions that might be useful in option pricing:

Corollary 1 ( [5]). A function $f$ satifying the growth condition (4.2) with Laplace transform $F(\lambda)$ is non-negative if and only if

$$
(-1)^{n} F^{(n)}(\lambda) \geq 0
$$

for all $n \geq 0$ and all $\lambda>\gamma$. 


\subsubsection{The Gaver-Stehfest methods}

Another inversion formula can also be obtained from the following arguments. Let

$$
I_{n}=\int_{0}^{\infty} \delta_{n}(t, u) f(t) d u
$$

where the functions $\delta_{n}(t, u)$ converge to the delta function as $n$ tends to infinity, thus

$$
\lim _{n \rightarrow \infty} I_{n}=f(t) .
$$

The right-hand side of equation (4.6) can be presented as some function multiplied by the $n$-th derivative of the Laplace transform of $f(t)$. For example the Post-Widder formula can be obtained from (4.6) letting

$$
\delta_{n}(t, u)=\left(\frac{n u}{t}\right)^{n} \frac{e^{-n u / t}}{(n-1) !} .
$$

Using similar arguments Gaver [16] suggested to choose the functions

$$
\delta_{n}(t, u)=\frac{(2 n) !}{n !(n-1) !} a\left(1-e^{-a u}\right)^{n} e^{-n a u}
$$

where $a=\ln (2 / t)$, which leads to

$$
f(t)=\lim _{n \rightarrow \infty} I_{n}(t)=\lim _{n \rightarrow \infty} \frac{(2 n) !}{n !(n-1) !} a \Delta^{n} F(n a) .
$$

It is similar to the Post-Widder formula, but instead of $F^{(n)}(\lambda)$ we have the $n$-th finite difference $\Delta^{n} F(\lambda)$. Still, the convergence of $I_{n}$ to $\mathrm{f}(\mathrm{t})$ is too slow. But Gaver showed that $\left(I_{n}-f(t)\right)$ can be expanded asymptotically in powers of $(1 / n)$, and Stehfest improved the Gaver's method [28] and presented an algorithm based on approximating $f(t)$ by the following sum

$$
a \sum_{n=1}^{N} K_{n} F(n a)
$$

where

$$
K_{n}=(-1)^{n+N / 2} \sum_{k=[(n+1) / 2]}^{\min (n, N / 2)} \frac{k^{N / 2}(2 k) !}{(N / 2-k) ! k !(k-1) !(n-k) !(2 k-n) !} .
$$

This resulting algorithm is called the Gaver-Stehfest algorithm. 


\subsubsection{The Kryzhnyi method}

In this work we also consider the method of inverse Laplace transform suggested by Kryzhnyi [21]. It was never implemented before to valuate options. Kryzhnyi [21] suggests that the algorithms, which are based on choosing different delta convergent sequences can be compared by analyzing the 'focusing' abilities of the numerical and the exact inverse transforms of $e^{\lambda t}$. Kryzhnyi considered

$$
F_{R}(\gamma+\lambda)=\int_{0}^{\infty} F(\gamma+\lambda u) \delta_{R}(u-1) d u,
$$

where $\lambda, \gamma$ are complex parameters and contructed series approximating the delta function $\delta_{R}(u-1) \rightarrow \delta(u-1)$ for $R \rightarrow \infty$ and thus $F_{R}(\gamma+\lambda) \rightarrow F(\gamma+\lambda)$ as $R \rightarrow \infty$. Note that the delta series can be selected such that the smoothing transformation (4.8) converges [21]. Let us note that this transformation (4.8) is used to obtain, using the Cauchy-Goursat Theorem, a formula for the analytic continuation by rotation allowing us to invert a real-valued Laplace transform by computing the Bromwich integral (4.4) in the complex plane.

Focusing abilities means how does the peakness of a delta approximating function is kept while increasing $t$. Of course this function flatterns with the time, it happens because the kernel of the integral (4.6) satisfies a scaling property.

Focusing on this qualitative characteristics Kryzhnyi developed another algorithm of approximating the original function from its Laplace transform. Firstly, he applied the Mellin transform to equation (4.1) (see Appendix for definitions) and got a solution in terms of the Mellin transform. which can be inverted after multiplying it by a suitable chosen regularization factor

$$
\delta_{R}(u-1)=\frac{\sin (R \ln u)}{\pi(u-1)} d u
$$

The result can be expressed by two equations

$$
\begin{gathered}
f_{R}=\int_{0}^{\infty} f(t u) \frac{\sqrt{u}}{u+1} \frac{\sin (R \ln u)}{u-1} d u, \\
f_{R}=\int_{0}^{\infty} F(u) \Pi(R, t u) d u,
\end{gathered}
$$

where $\gamma$ is a regularization parameter and $R(\gamma) \rightarrow \infty$, while $\gamma \rightarrow 0$.

Here, instead of some number $N$, after which we terminate the computation we have a value of some function $R$ in point $\gamma$.

After some generalization we have

$$
\Pi(R, u)=\frac{1}{\pi \varphi(1)} \mathcal{L}^{-1}\left[\frac{\sin (R \ln p)}{p-1} \varphi(p)\right],
$$


where $\varphi(u)$ is an arbitrary continuous function $\varphi(1) \neq 0$. From this equation follows that various kernels can be constructed in this way by choosing the function $\varphi(u)$. However, we can choose $\varphi(u)$ in such a way that the kernel can be expressed analytically using known transforms from tables.

Actually this approach by Kryzhnyi will be more tunable for different types of problems, because we can vary the regularization parameter $\gamma$ and choose different functions $\varphi(u)$. There are some limitations on $R$ as pointed out by Kryzhnyi [21]:

- $\lim _{R \rightarrow \infty} \Pi(R, x)$ does not exist,

- for the fixed precision arithmetic the value of parameter $R>0$ cannot be increased infinitely without loss of accuracy, which is explained by the next limitation,

- the optimal value of the parameter $R$ is close to a linear function of number $n$ of correct digits in the input data: $n / 2<R_{\mathrm{opt}}<n$.

Nevertheless, the technic of choosing these parameters $R$ and $\gamma$ is rather complicated.

Furthermore, the approach of Kryzhnyi allows the see all the methods for the Laplace inversion in a unified seeting, e.g. the method of Gaver (4.7) can be obtained by choosing the regularization

$$
\delta_{n}(u)=\frac{(2 n) ! \ln 2}{n !(n-1) !}\left(2^{-u}-2^{-2 u}\right)^{n} .
$$

\section{The analytical expressions in transformed variables}

\subsection{Transformed option values}

Next we present Kimura's results [20] in transformed variables from equation (3.4). For convenience the direction of time is reverted by the change of the variable $\tau=$ $T-t$ and defining $\tilde{c}(\tau, S ; q)=c(T-\tau, S ; q)=c\left(t, S_{t} ; q\right)$ and $\underline{\tilde{S}}_{\tau}=S_{T-\tau}=S_{t}$ for $\tau \leq 0$. The Laplace-Carson transform of this variables follows from Definition 2

$$
\begin{gathered}
\mathcal{c}^{*}(\lambda, S ; q)=\mathcal{L C}\{\tilde{\mathcal{C}}(\tau, S ; q)\} \equiv \lambda \int_{0}^{\infty} e^{-\lambda \tau} \tilde{\mathcal{C}}(\tau, S ; q) d \tau, \\
\underline{S}^{*}(\lambda)=\mathcal{L C}\{\underline{\tilde{S}}(\tau ; q)\} \equiv \lambda \int_{0}^{\infty} e^{-\lambda \tau} \underline{\tilde{S}}_{\tau} d \tau .
\end{gathered}
$$

Again, the Laplace-Carson transform is preferred to the Laplace transform because the constant values do not change the transformation and the Laplace-Carson approach generates simpler formulas for our problem. Applying the Laplace-Carson transform to the inhomogeneous PDE (3.4) we get an inhomogeneous ODE of the same order. 
For solving this type of ODE we need to solve the corresponding homogeneous ODE, so it makes sense first to consider the transformation of the original Black-Scholes PDE for the vanilla options, where the parameter $q$ is absent.

Lemma 1. Let $c^{*}(\lambda, S)=\mathcal{L C}\{\tilde{c}(\tau, S)\}$ define a Laplace-Carson transform of a value of a vanilla call option with the reversed time. Then

$$
c^{*}(\lambda, S)= \begin{cases}\frac{K}{\theta_{1}-\theta_{2}} \frac{\lambda}{\lambda+\delta}\left(1-\frac{r-\delta}{\lambda+r} \theta_{2}\right)\left(\frac{S}{K}\right)^{\theta_{1}}, & \text { if } S<K, \\ \frac{K}{\theta_{1}-\theta_{2}} \frac{\lambda}{\lambda+\delta}\left(1-\frac{r-\delta}{\lambda+r} \theta_{2}\right)\left(\frac{S}{K}\right)^{\theta_{1}}+\frac{\lambda S}{\lambda+\delta}-\frac{\lambda K}{\lambda+r}, & \text { if } S \geq K,\end{cases}
$$

where $\theta_{1}$ and $\theta_{2}$ depend on $\lambda$ and are real roots of the quadratic equation

$$
\frac{1}{2} \sigma^{2} \theta^{2}+\left(r-\delta-\frac{1}{2} \sigma^{2}\right) \theta-(\lambda+r)=0
$$

It can be seen that when putting $\theta=1$ and $\theta=0$ we get negative values on the left hand side of equation (5.2). This means that both roots are outside the interval $(0,1)$, so we numerate it in such a way that $\theta_{1}>1$ and $\theta_{2}<0$.

Proof: The original proof can be found in [20]. After changing variables the BlackScholes PDE reads

$$
-\frac{\partial \tilde{c}}{\partial \tau}+(r-\delta) S_{\tau} \frac{\partial \tilde{c}}{\partial S}+\frac{1}{2} \sigma^{2} S_{\tau}^{2} \frac{\partial^{2} \tilde{c}}{\partial S^{2}}-r \tilde{c}=0, \quad S>0,
$$

supplied with the boundary conditions

$$
\lim _{S \rightarrow+0} \tilde{c}(t, S)=0, \quad \lim _{S \rightarrow \infty} \frac{d \tilde{c}}{d S}<\infty,
$$

and the initial condition

$$
\tilde{c}(0, S)=(S-K)^{+} .
$$

After transforming equation (5.3) we obtain a corresponding ODE

$$
\frac{\sigma^{2}}{2} S^{2} \frac{d^{2} c^{*}}{d S^{2}}+(r-\delta) S \frac{d c^{*}}{d S}-(\lambda+r) c^{*}+\lambda(S-K)^{+}=0, \quad S>0,
$$

with the boundary conditions

$$
\lim _{S \rightarrow+0} c^{*}(\lambda, S)=0, \quad \lim _{S \rightarrow \infty} \frac{d c^{*}}{d S}<\infty .
$$

Equation (5.4) is a linear homogeneous ODE of Euler type and can be reduced to a linear ODE with constant coefficients by substituting $S=e^{y}$ (Euler transformation) and solved easily yielding (5.1). 
Theorem 2 ([20]). If $S>\underline{S}^{*}$, then

$$
c^{*}(\lambda, S ; q)=c^{*}(\lambda, S)+\frac{q}{\lambda+r} \frac{\theta_{1}}{\theta_{1}-\theta_{2}}\left(\frac{S}{\underline{S}^{*}}\right)
$$

and $c^{*}(\lambda, S ; q)=0$ otherwise. The stopping boundary is given by

$$
\underline{S}^{*}(\lambda)=\left[\frac{2(\lambda+\delta) q}{\lambda\left(1-\theta_{2}\right) K \sigma^{2}}\right]^{-\theta_{1}} K
$$

It is straightforward that the solution for this equation is a sum of solutions for the homogeneous equation and a particular solution of the inhomogeneous equation. It can be easily seen that the second part of the formula for $c^{*}(\lambda, S ; q)$, without $c^{*}(\lambda, S)$ is a solution for the corresponding inhomogeneous ODE.

Additionally, the same approach can be used for proceeding the solution for the put case. The result can be formulated by the following theorem.

Theorem 3 ( [20]). If $S<\bar{S}^{*}$, then

$$
p^{*}(\lambda, S ; q)=p^{*}(\lambda, S)+\frac{q}{\lambda+r} \frac{\theta_{2}}{\theta_{1}-\theta_{2}}\left(\frac{S}{\bar{S}^{*}}\right)^{\theta_{1}},
$$

and $p^{*}(\lambda, S ; q)=0$ otherwise. With

$$
p^{*}(\lambda, S)= \begin{cases}\frac{K}{\theta_{1}-\theta_{2}} \frac{\lambda}{\lambda+\delta}\left(1-\frac{r-\delta}{\lambda+r} \theta_{2}\right)\left(\frac{S}{K}\right)^{\theta_{1}}, & \text { if } S \geq K, \\ \frac{K}{\theta_{1}-\theta_{2}} \frac{\lambda}{\lambda+\delta}\left(1-\frac{r-\delta}{\lambda+r} \theta_{2}\right)\left(\frac{S}{K}\right)^{\theta_{1}}+\frac{\lambda S}{\lambda+\delta}-\frac{\lambda K}{\lambda+r}, & \text { if } S<K .\end{cases}
$$

The stopping boundary is given by

$$
\bar{S}^{*}(\lambda)=\left[\frac{2(\lambda+\delta) q}{\lambda\left(\theta_{1}-1\right) K \sigma^{2}}\right]^{-\theta_{1}} K .
$$

\subsection{Transformed Greeks}

In Section 3.2 we mentioned the decomposition of the total premium of the installment option. This decomposition of the option in a vanilla call option and an American compound option was shown by Kimura [20] to be very valuable when trying to approximate the installment options Greeks. We have

$$
c\left(t, S_{t} ; q\right)+K_{t}=c\left(t, S_{t}\right)+P_{c}\left(t, S_{t} ; q\right),
$$

with

$$
K_{t}=\frac{q}{r}\left(1-e^{-r(T-t)}\right) .
$$


Using the integral representation (3.5) we obtain

$$
K_{t}-P_{c}\left(t, S_{t} ; q\right)=q \int_{t}^{T} e^{-r(u-t)} \Phi\left(d_{-}\left(S_{t}, \underline{S}_{u}, u-t\right)\right) d u .
$$

Substituting $\Phi(x)=1-\Phi(-x)$ we get an integral representation for the American compound option

$$
P_{c}\left(t, S_{t} ; q\right)=q \int_{t}^{T} e^{-r(u-t)} \Phi\left(-d_{-}\left(S_{t}, \underline{S}_{u}, u-t\right)\right) d u .
$$

Due to the linearity of the Laplace-Carson transform we get for the time-reversed values

$$
\mathcal{L C}\left\{\tilde{c}\left(t, \tilde{S}_{t} ; q\right)\right\}+\mathcal{L C}\left\{\tilde{K}_{t}\right\}=\mathcal{L C}\left\{\tilde{c}\left(t, \tilde{S}_{t}\right)\right\}+\mathcal{L C}\left\{\tilde{P}_{c}\left(\tau, \tilde{S}_{;} q\right)\right\}
$$

From Theorem 2 we see that

$$
P_{c}^{*}\left(t, S_{t} ; q\right)-K_{t}^{*}=\frac{q}{\lambda+r} \frac{\theta_{1}}{\theta_{1}-\theta_{2}}\left(\frac{S}{\bar{S}^{*}}\right)^{\theta_{2}}-\frac{q}{\lambda+r} .
$$

Here, the inverse Laplace-Carson transform of the term $\frac{q}{\lambda+r}$ can be computed analytically

$$
\mathcal{L C}^{-1}\left\{\frac{q}{\lambda+r}\right\}=q \int_{0}^{\tau} e^{-r u} d u=\frac{q}{r}\left(1-e^{-r(T-t)}\right)=K_{t},
$$

thus for the transformed value of an American put on a call we have

$$
P_{c}^{*}\left(t, S_{t} ; q\right)=\frac{q}{\lambda+r} \frac{\theta_{1}}{\theta_{1}-\theta_{2}}\left(\frac{S}{\bar{S}^{*}}\right)^{\theta_{2}}
$$

Hence the Greeks of the continuous installment call option can be expressed by Greeks of the vanilla call and Greeks of the American put on a vanilla call with a floating strike price $K_{t}$ :

$$
\begin{aligned}
\Delta_{c(t, S ; q)} & =\frac{\partial c}{\partial S}=\Delta_{c t, S}+\mathcal{L C}^{-1}\left\{\Delta_{P_{c}^{*}}\right\}, \\
\Gamma_{c(t, S ; q)} & =\frac{\partial^{2} c}{\partial S^{2}}=\Gamma_{c t, S}+\mathcal{L C}^{-1}\left\{\Gamma_{P_{c}^{*}}\right\}, \\
\Theta_{c(t, S ; q)} & =-\frac{\partial c}{\partial \tau}=\Theta_{c t, S}+q e^{-r \tau} \mathcal{L C}^{-1}\left\{\Theta_{P_{c}^{*}}\right\} .
\end{aligned}
$$

Now using (5.9) we find explicit formulas for the transformed values of American compound option greeks, 


$$
\begin{aligned}
\Delta_{P_{c}^{*}}=\mathcal{L C}\left\{\frac{\partial P_{c}^{*}}{\partial S}\right\} & =\frac{\partial P_{c}^{*}}{\partial S}, \\
\Gamma_{P_{c}^{*}}=\mathcal{L C}\left\{\frac{\partial^{2} P_{c}^{*}}{\partial S^{2}}\right\} & =-\frac{\partial^{2} P_{c}^{*}}{\partial S^{2}}, \\
\Theta_{P_{c}^{*}}=-\mathcal{L C}\left\{\frac{\partial P_{c}^{*}}{\partial \tau}\right\} & =-\lambda\left(P_{c}^{*}(\lambda, S ; q)-P_{c}^{*}(0, S ; q)\right) \\
& =-\lambda P_{c}^{*}(\lambda, S ; q) .
\end{aligned}
$$

Using the same arguments for the installment put case we obtain

$$
\begin{aligned}
\Delta_{p(t, S ; q)} & =\frac{\partial p}{\partial S}=\Delta_{p t, S}+\mathcal{L C}^{-1}\left\{\Delta_{P_{p}^{*}}\right\} \\
\Gamma_{p(t, S ; q)} & =\frac{\partial^{2} p}{\partial S^{2}}=\Gamma_{p t, S}+\mathcal{L C}^{-1}\left\{\Gamma_{P_{p}^{*}}\right\} \\
\Theta_{p(t, S ; q)} & =-\frac{\partial p}{\partial \tau}=\Theta_{p t, S}+q e^{-r \tau} \mathcal{L C}^{-1}\left\{\Theta_{P_{p}^{*}}\right\} .
\end{aligned}
$$

Correspondingly, we have

$$
\begin{aligned}
\Delta_{P_{p}^{*}}=\mathcal{L C}\left\{\frac{\partial P_{p}^{*}}{\partial S}\right\} & =\frac{\partial P_{p}^{*}}{\partial S} \\
\Gamma_{P_{p}^{*}}=\mathcal{L C}\left\{\frac{\partial^{2} P_{p}^{*}}{\partial S^{2}}\right\} & =-\frac{\partial^{2} P_{p}^{*}}{\partial S^{2}} \\
\Theta_{P_{p}^{*}}=-\mathcal{L C}\left\{\frac{\partial P_{p}^{*}}{\partial \tau}\right\} & =-\lambda\left(P_{p}^{*}(\lambda, S ; q)-P_{p}^{*}(0, S ; q)\right) \\
& =-\lambda P_{p}^{*}(\lambda, S ; q) .
\end{aligned}
$$

\section{Results}

We developed a set of Matlab functions for valuing continuous installment options and its Greeks via the inverse Laplace transform methods. The algorithm is based on results of Kimura [20], in which the author uses two algorithms for the inverse Laplace transform: the Euler summation [25] and the Gaver-Stehfest method. In our case we use the Euler, the Gaver-Stehfest and also the Kryzhnyi algorithms, described in Section 4.

Our algorithm of the continuous installment option valuing consists of two numerical procedures. It is finding the stopping boundary and the numerical integration of the integral in (3.5) or (3.6). The difference in comparison to Kimura's algorithm is that we use the standard quadrature Matlab routine quad, which uses the Simpson 

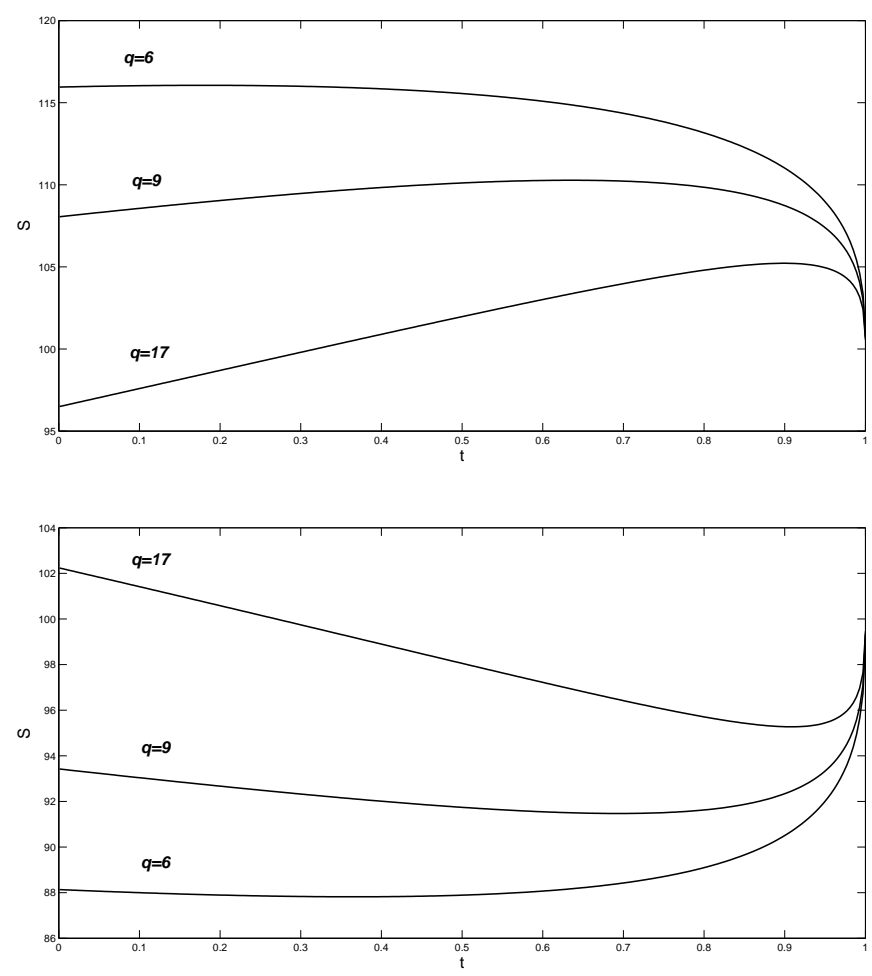

Figure 2: Stopping boundaries for put and call with $T=1, t=0, \delta=0.03, r=0.02$.

formula for the integration and determines integration nodes automatically and then evaluates the stopping boundary in each node.

The results of approximation of the stopping boundaries for the put and the call cases and its sensitivity to the installment rate $q$ and the dividend yield $\delta$ are presented in Figure 2 and Figure 3. From the Figures it can be seen that in dependence on parameters $q$ and $\delta$, the stopping boundary can be either a monotonic or non-monotonic function, unlike the exercise boundaries of the American style options. This nonmonotonic behavior also appears in some types of Asian options and draws great interest of researchers.

The values of the continuous installment options obtained by our Matlab programme are given in the last three columns of Tables 1 and 2. The results of the developed Euler method are compared to the results taken from [20]. Kimura also notes that every value obtained by the Gaver-Stehfest algorithm is smaller than the values obtained by the Euler summation. In his results, these values differ significantly, which caused the author to mistrust to the Gaver-Stehfest algorithm. But as for our results, it can be seen from the tables that all three algorithms produce very close values. In Figure 4 you can see a 3D plot of the call and the put values in dependence on time and asset price.

The values for different Greeks are presented in Figures 5, 6, 7. Actually not all 

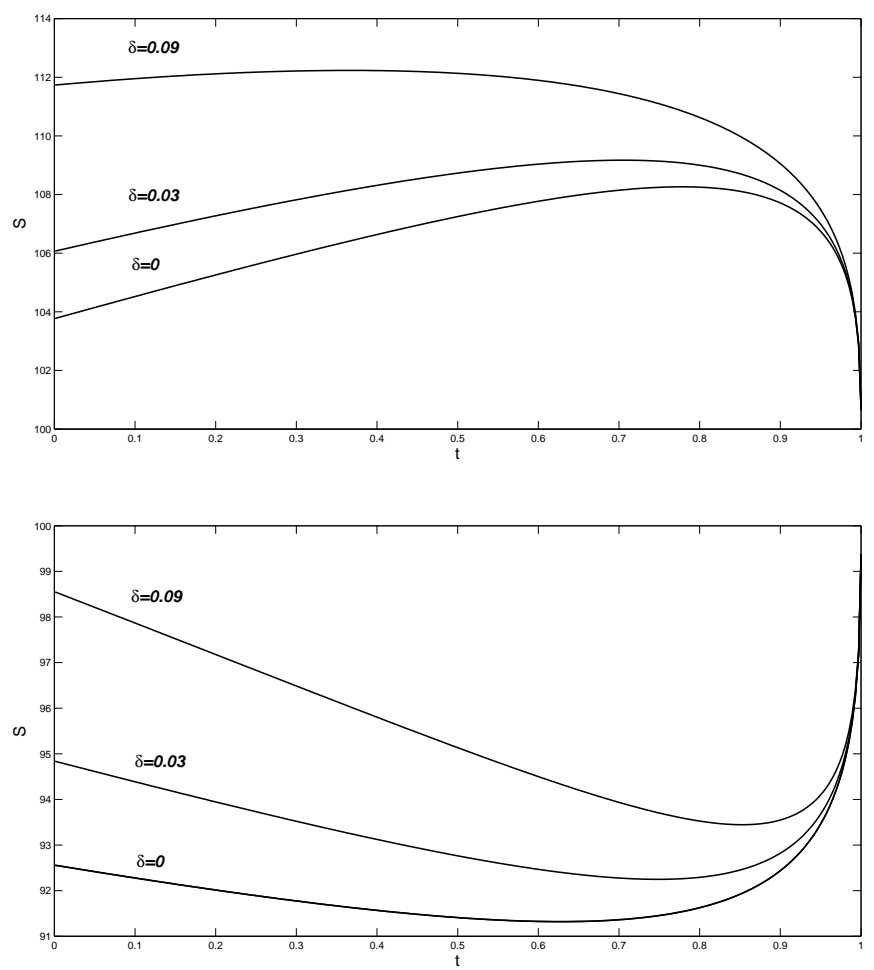

Figure 3: Stopping boundaries for the put and the call with $T=1, t=0, q=10$, $r=0.02$.

values for Greeks presented here make sense. We can only evaluate Greeks if we are above the stopping boundary in the call case and below a stopping boundary in the put case. In Figure 6 you can see an unexpected blow up of the put gamma in case of $q=15$. In the figure black markers define the value of the stopping boundary in each case. So this unexpected behavior has no mean for us, because it happens after reaching the stopping boundary.

Kimura [20] noticed that the Gaver-Stehfest method behaves badly for valuing Greeks when the position is out of the money, but we did not notice that. The results for both used inverse methods look quite reasonable in the whole region where the stopping boundary is not reached, even for a time very close to expiration.

Trying to compare the Gaver-Stehfest and the Kryzhnyi algorithms we used a comparison method, proposed by Kryzhnyi [21]. The method is based on inverting the function $e^{\lambda x}$, which analytical inverse transform is the delta function. The better the algorithm approximates the delta function while inverting $e^{\lambda x}$ and preserves its peakness while increasing $t$, the better it will approximate other functions too. In Figure 8 you can see the results of the reconstructing the delta function. The Kryzhnyi method shows more peaked values and more slowly flatterns with time, therefore Kryzhnyi method reconstructs the function much better in the area of a peak. 


\begin{tabular}{|c|c|c|c|c|c|}
\hline $\mathrm{q}$ & $\mathrm{S}$ & Euler-based (Kimura) & Euler-based & Gaver-Stehfest & Kryzhnyi \\
\hline 1 & 95 & 3.7071 & 3.7072 & 3.7072 & 3.7069 \\
& 105 & 8.3994 & 8.3995 & 8.3995 & 8.3993 \\
& 115 & 14.8530 & 14.8531 & 14.8531 & 14.8531 \\
\hline 3 & 95 & 2.2280 & 2.2283 & 2.2283 & 2.2266 \\
& 105 & 6.6385 & 6.6388 & 6.6388 & 6.6379 \\
& 115 & 12.9687 & 12.9690 & 12.9690 & 12.9686 \\
\hline 6 & 95 & 0.6754 & 0.6761 & 0.6761 & 0.6703 \\
& 105 & 4.2745 & 4.2752 & 4.2752 & 4.2723 \\
& 115 & 10.2533 & 10.2540 & 10.2540 & 10.2527 \\
\hline
\end{tabular}

Table 1: Values of call with $t=0, T=1, K=100, r=0.03, \delta=0.05, \sigma=0.2$ computed by different algorithms.

\begin{tabular}{|c|c|c|c|c|c|}
\hline $\mathrm{q}$ & $\mathrm{S}$ & Euler-based (Kimura) & Euler-based & Gaver-Stehfest & Kryzhnyi \\
\hline 1 & 85 & 16.9438 & 16.9439 & 16.9439 & 16.9439 \\
& 95 & 10.3046 & 10.3047 & 10.3047 & 10.3047 \\
& 105 & 5.5703 & 5.5703 & 5.5704 & 5.5705 \\
\hline 3 & 85 & 15.0001 & 15.0007 & 15.0008 & 15.0009 \\
& 95 & 8.4283 & 8.4285 & 8.4286 & 8.4289 \\
& 105 & 3.8486 & 3.8489 & 3.8489 & 3.8497 \\
\hline 6 & 85 & 12.1253 & 12.1259 & 12.1259 & 12.1263 \\
& 95 & 5.7647 & 5.7652 & 5.7652 & 5.7666 \\
& 105 & 1.7010 & 1.7018 & 1.7018 & 1.7051 \\
\hline
\end{tabular}

Table 2: Values of put with $t=0, T=1, K=100, r=0.03, \delta=0.05, \sigma=0.2$ computed by different algorithms.

When reconstructing monotonic functions from its Laplace transform both Kryzhnyi and the Gaver-Stehfest methods show good results, but when dealing with a damped oscillating function it occurs that the Gaver-Stehfest algorithm cannot compete with the Kryzhnyi method. In Figure 9 we see that the curve, produced by the Stehfest algorithm flatterns much faster than the one produced by the Kryzhnyi method. In our case it is difficult to say which of the methods is more precise because we are reconstructing the non-oscillating functions, but as for computational costs it is more convenient to use the Gaver-Stehfest algorithm. 

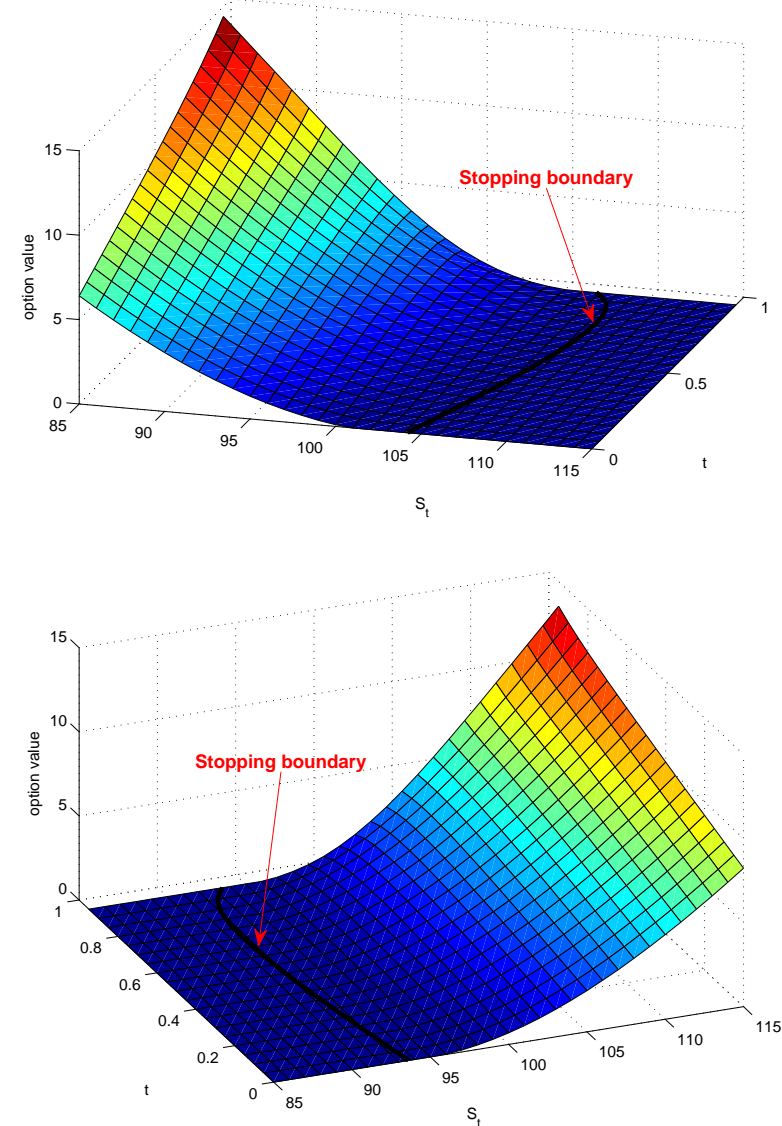

Figure 4: The option value for the put and the call with $T=1, t=0, \delta=0.03, r=0.02$, $q=10$ and stopping boundaries. 

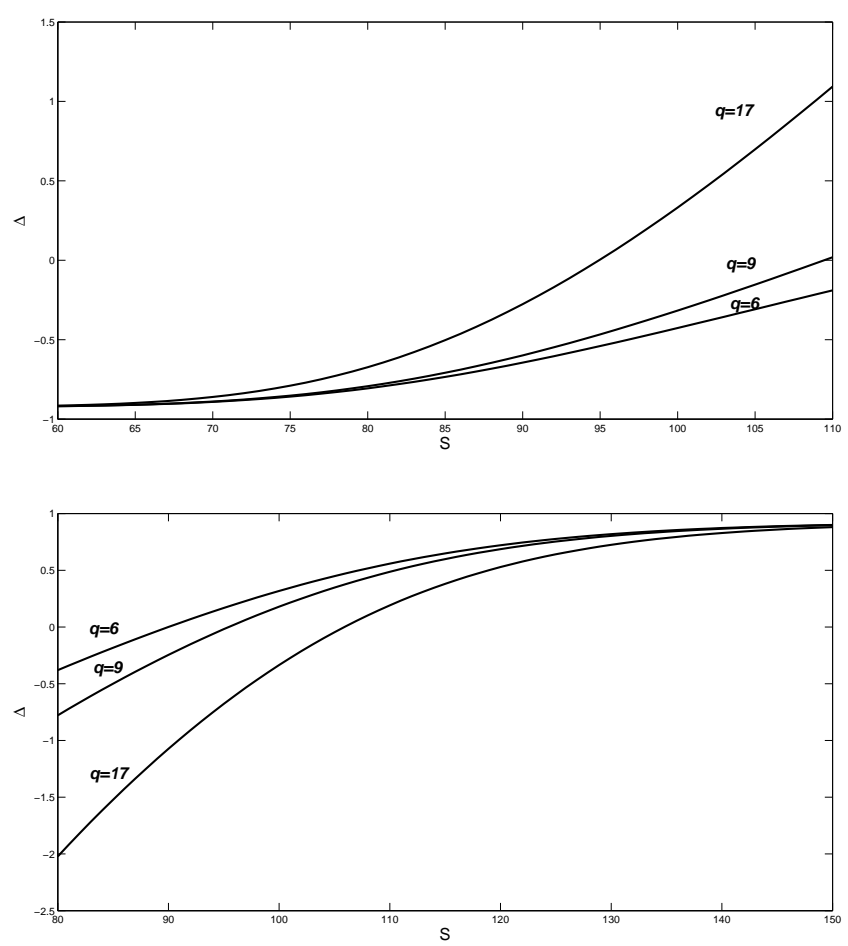

Figure 5: The Delta value for the put and the call, in dependence on $q$ where $q=6,9$, 17 with $T=1, t=0, r=0.02, \delta=0.04$.

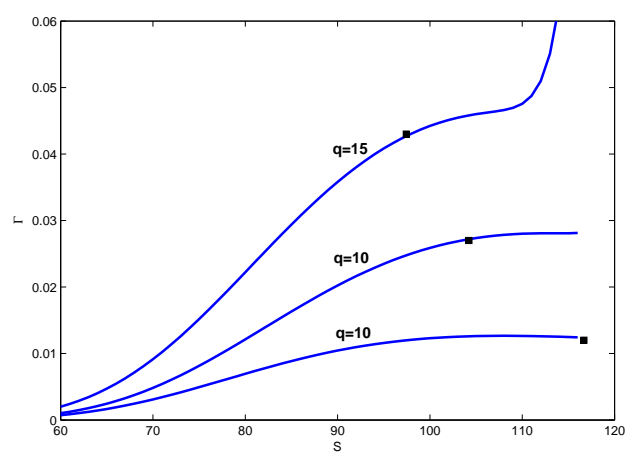

(a) Put case

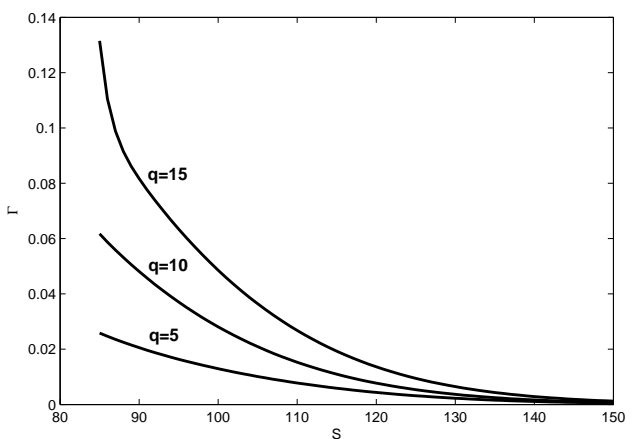

(b) Call case

Figure 6: The Gamma value for the put and the call, in dependence on $q$ where $q=5$, 10,15 with $T=1, t=0, r=0.02, \delta=0.04$. 


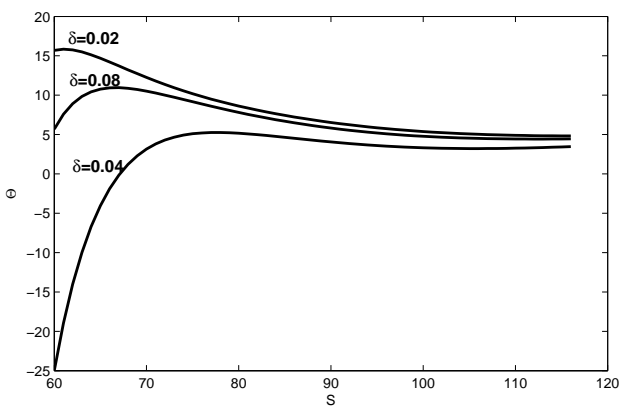

(a) Put case

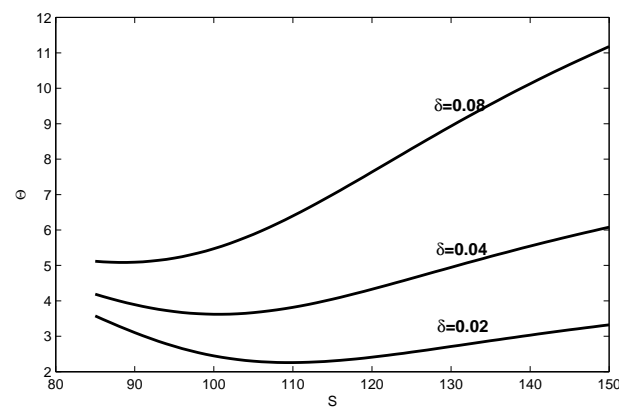

(b) Call case

Figure 7: The Theta value for the put and the call, in dependence on $\delta$ where $\delta=0.08$, $0.04,0.02$ with $T=1, t=0, r=0.02, \delta=0.04$.

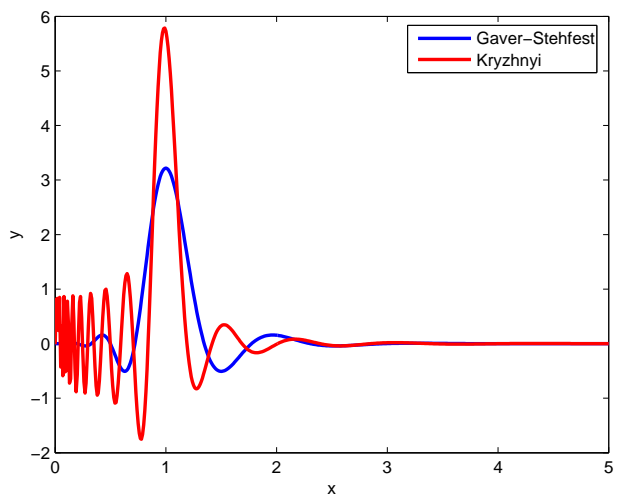

(a) $t=1$

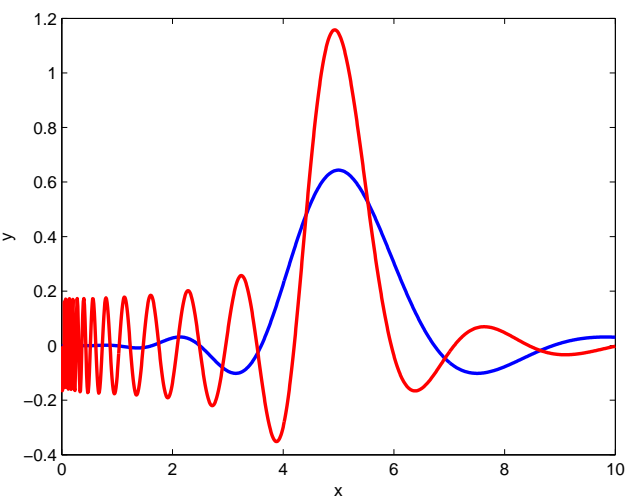

(b) $t=5$

Figure 8: The reconstruction of the delta function by Stehfest and Kryzhnyi algorithms. 


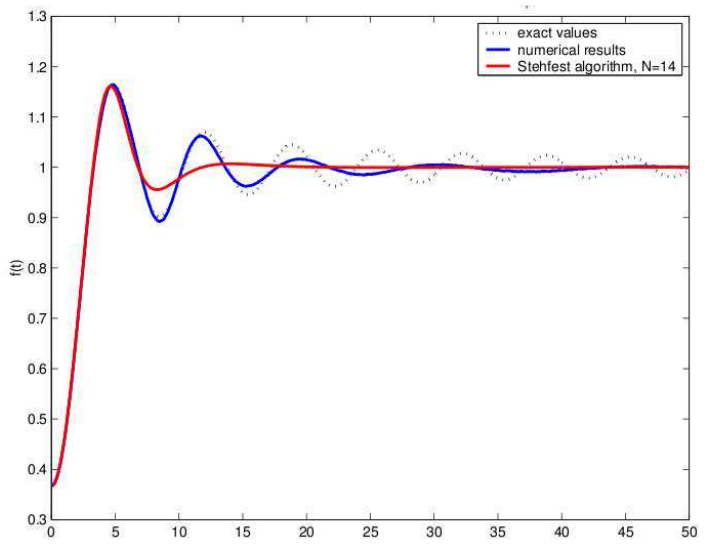

Figure 9: The reconstruction of the damped oscillating function by Stehfest and Kryzhnyi algorithms. The dashed line - the exact values, the red line - values obtained by the Gaver-Stehfest method, the blue line - values obtained by the Kryzhnyi method. 


\section{Conclusions}

We considered the valuation of the European continuous installment options, where we faced the stopping boundary problem. To simplify the stopping boundary expression we used the Laplace-Carson transformation as it was proposed by Kimura [20]. The computation of the transformed values is followed by the inverse Laplace transformation procedure.

We mainly consider two methods of the inverse Laplace transformation: the GaverStehfest method that was used by Kimura to valuate the continuous installment options and the Kryzhnyi method that was never applied before for the valuation of options. Eventually, we developed the program code for Matlab to compute the stopping boundaries, values and Greeks of the European continuous installment options, implementing above methods.

Contrary to Kimura's conclusions, who relies on the Euler method only as the most precise one, our numerical computation shows that all three methods - the Euler, the Gaver-Stehfest and the Kryzhnyi - produce very close results. We conclude that there is no reason to mistrust any of them. They all can be used to value continuous installment options, however considering the computational costs we would rather suggest to use the Euler method as it is the fastest one of the considered methods.

In our future work we will consider more alternative algorithms for the numerical inverse Laplace transform, like e.g. a modification of the original (Gaver-Stehfest) algorithm, the BigNumber-Stehfest algorithm [1] or the Fourier Series Expansion method $[8,14]$ with its quotient-difference acceleration algorithm by De Hoog et al. [18] (the later method is available as Matlab routine invlap). Each of these methods has the ability to invert properly certain classes of Laplace space functions, e.g. the Fourier Series method is suitable for the long time integration of dissipative equations and thus we expect that it is also a good candidate in our application to the parabolic Black-Scholes equation.

Moreover, based on the numerical inverse Mellin transform method of Migneron and Narayanan [24] we will derive on a fully discrete way an efficient three term recursion algorithm to compute numerically the inverse Mellin transform, hereby using the ideas proposed by Ehrhardt in [15]. The resulting method will be applied to option pricing problems.

\section{Acknowledgments}

The authors are grateful to Vladimir Kryzhnyi for providing the program code of his novel method. Furthermore, the authors would like to thank professor Kimura for his kind cooperation and to Ljudmila Bordag of Halmstad University for her hospitality. 


\section{A Appendix: The Mellin Transformation}

Definition 3 (Mellin-transformation [27]). The Mellin-transformation of a local integrable function $f(t)$ of $(0, \infty)$ is defined as follows

$$
\mathcal{M}[f ; \lambda]=\hat{f}(\lambda)=\int_{0}^{\infty} f(t) t^{\lambda-1} d t,
$$

if the integral converges.

We remark that this integral converges absolutely, if for each $\epsilon>0$

$$
f(t)= \begin{cases}\mathrm{O}\left(t^{-a-\epsilon}\right) & t \rightarrow 0+ \\ \mathrm{O}\left(t^{-b+\epsilon}\right) & t \rightarrow \infty\end{cases}
$$

holds with $a<b$. I.e. the Mellin-transformation (A.1) exists on a vertical strip in the $\lambda$ plane, where the bounds results from the asymptotic behaviour of $f(t)$ for $t \rightarrow 0+$ and $t \rightarrow \infty$. The Mellin transform defines an analytic function on the strip $a<\operatorname{Re}(\lambda)<b$.

The Mellin-transformation can be derived from the Laplace-transformation

$$
\mathcal{L}[g ; \lambda]=\int_{-\infty}^{+\infty} g(\tau) e^{-\lambda \tau} d \tau
$$

with the aid of the transformation of variables $\tau:=-\ln t$. Here we set $f(t) \equiv$ $g(-\log t)$. Using the same transformation of variables one arrives from the Laplace inversion at the inverse Mellin transformation.

Definition 4 (inverse Mellin-transformation).

$$
\mathcal{M}^{-1}[\hat{f}(\lambda) ; t]=f(t)=\frac{1}{2 \pi i} \int_{c-i \infty}^{c+i \infty} t^{-\lambda} \hat{f}(\lambda) d \lambda
$$

for $a<c<b$. (A.2) exists for all $t \geq 0$, where $f(t)$ is continuous. For a proof we refer to [27].

\section{References}

[1] J. Abate and P.P. Valkó, Multi-precision Laplace transform inversion, Int. J. Numer. Meth. Engng, 60 (2004), 979-993.

[2] G. Alobaidi, R. Mallier and A.S. Deakin, Laplace transforms and installment options, Math. Models Meth. Appl. Sci, 8 (2004), 1167-1189.

[3] H. Bateman, Two systems of polynomials for the solution of Laplace's Integral Equation, Duke Math. J, 2 (1936), 569-577.

[4] H. Ben-Ameur, M. Breton and P. Francois, A dynamic programming approach to price installment options, European J. Oper. Research, 169 (2006), 667-676.

[5] K. Bryan, Elementary Inversion of the Laplace Transform, 2006, http://www . rose-hulman.edu/ bryan/invlap.pdf 
[6] P. Ciurlia and I. Roko, Valuation of American continuous-installment options, Comput. Econ, 25 (2005), 143-165.

[7] A. Cohen, Numerical Methods for Laplace Transform Inversion, Springer, New York, 2007, pp. 23-42, 141-146.

[8] K.S. Crump, Numerical Inversion of Laplace Transforms Using a Fourier Series Approximation, Journal of the ACM, 23 (1976), 89-96.

[9] B. Davies and B. Martin, Numerical inversion of the Laplace transform: a survey and comparison of methods, J. Comput. Phys, 33 (1979), 1-32.

[10] M. Davis, W. Schachermayer and R. Tompkins, Pricing, No-arbitrage Bounds and Robust Hedging of Installment Options, Quant. Finance, 1 (2001), 597-610.

[11] M. Davis, W. Schachermayer and R. Tompkins, Installment options and static hedging, J. Risk Finance, 3 (2002), 46-52.

[12] A.R. Dixit and R.S. Pindyck, Investment Under Uncertainty, Princeton University Press, 1994.

[13] C.W. Dong, A regularization method for numerical inversion of the Laplace transform, SIAM J. Numer. Anal, 30 (1993), 759-773.

[14] D.G. Duffy, On the numerical inversion of Laplace transforms: Comparison of three new methods on characteristic problems from applications, ACM Trans. Math. Soft, 19 (1993), 333-359.

[15] M. Ehrhardt, Discrete Artificial Boundary Conditions, Ph.D. Thesis, Technische Universität Berlin, 2001.

[16] D.P. Gaver, Observing Stochastic Processes and approximate Transform Inversion, Operational Res, 14 (1966), 444-459.

[17] S. Griebsch, C. Kühn and U. Wystup, Instalment Options: a Closed-Form Solution and the Limiting Case, Mathematical Control Theory and Finance, Springer, Heidelberg, 2008, pp. 211-229.

[18] F.R. de Hoog, J.H. Knight and A.N. Stokes, An improved method for numerical inversion of Laplace transforms, SIAM J. Sci. Stat. Comp, 3 (1982), 357-366.

[19] F. Karsenty and J. Sikorav, Installment plan, Over the Rainbow, Risk magazin (1993), 36-40.

[20] T. Kimura, Valuing Continuous-Installment Options, European J. Oper. Res, 201 (2010), 222-230.

[21] V. Kryzhnyi, Numerical inversion of the Laplace transform: analysis via regularized analytic continuation, Inverse Problems, 22 (2006), 579-597.

[22] V. Kryzhnyi, Freeware DLL for Matlab at http://www. laplacetransform. org/

[23] C.D. MacRae, The Employee Stock Option: An Installment Option, 2008, Available at SSRN: http: //ssrn. com/abstract=1286928.

[24] R. Migneron and K.S.S. Narayanan, Numerical Inversion of Mellin Moments and Laplace Transforms, Comput. Phys. Commun, 49 (1988), 457-463.

[25] C. O'Cinneide, Euler summation for Fourier series and Laplace transform inversion, Stochastic Models, 13 (1997), 315-337.

[26] E.L. Post, Generalized differentiation, Trans. Am. Math. Soc, 32 (1930), 723-781.

[27] I.H. Sneddon, The Use of Integral Transforms, McGraw-Hill Book Company, 1972.

[28] H. Stehfest, Algorithm 368: Numerical inversion of Laplace Transform, Comm. ACM, 13 (1970), 47-49.

[29] L. Thomassen and M. Van Wouwe, The Influence of a Stochastic Interest Rate on the nfold Compound option, Statistics for Industry and Technology, Springer-Verlag, Berlin, 2004, pp. 343-353. 
[30] P.P. Valkó and J. Abate, Comparison of sequence accelerators for the Gaver method of numerical Laplace transform inversion, Comput. Math. Appl, 48 (2004), 629-636.

[31] J.A.C. Weideman, Algorithms for parameter selection in the Weeks method for inverting the Laplace transform, SIAM J. Sci. Comput, 21 (1999), 111-128.

[32] D.V. Widder, The inversion of the Laplace integral and the related moment problem, Trans. Math. Soc, 36 (1934), 107-200. 